\title{
Damping of injection perturbations at the European Synchrotron Radiation Facility
}

\author{
S. White, J. Chavanne, M. Dubrulle, G. Le Bec, E. Plouviez, P. Raimondi, and B. Roche \\ European Synchrotron Radiation Facility, 71, avenue des Martyrs, \\ CS 40220, 38043 Grenoble Cedex 9, Grenoble, France
}

(Received 5 December 2018; published 27 March 2019)

\begin{abstract}
Top-up operation was recently introduced at the European Synchrotron Radiation Facility (ESRF) to provide constant beam current to the beam line users. This mode of operation involves frequent injections that can be the source of significant perturbations on the stored beam. Beam lines running experiments with a timescale longer than the injection period may be impacted by such perturbations. The ESRF storage ring injection systems and layout were not designed to allow for transparent injection, i.e., without visible perturbations on the stored beam. These perturbations are driven directly or indirectly by rapidly pulsing elements, the effect of which cannot be corrected by standard methods such as feedback loops. Major efforts were put into the development of mitigation measures at the ESRF. Depending on the source of perturbation different approaches were used. They all contribute to the achievement of transparent injections and unperturbed continuous data acquisition for the beam lines. After a review of the ESRF injection systems, these developments and the resulting reduction of the injection perturbations are described in this paper.
\end{abstract}

DOI: $10.1103 /$ PhysRevAccelBeams.22.032803

\section{INTRODUCTION}

The ESRF $6 \mathrm{GeV}$ storage ring lattice consists of 32 double bend achromat (DBA) cells with alternating high- $\beta$ and low- $\beta$ straight sections where the insertion devices are located. One of the high- $\beta$ straight sections is dedicated to injection, where off-axis injection and accumulation is possible thanks to the large dynamic aperture at the injection point. This straight section and the neighboring arcs are hosting all the injection elements. The beam is transported from the ramped synchrotron booster to the storage ring using the TL2 transfer line. At the end of TL2, an in-vacuum septum (S3) and two in-air septa powered in series $(\mathrm{S} 1 / 2)$ are used to steer the injected beam on a trajectory parallel to the stored beam orbit. In the storage ring, four pulsed kickers powered independently are used to generate the injection bump. A similar layout with a single kicker is found at the booster extraction [1].

Figure 1 shows the ESRF injection region layout, optics functions and the injection bump. The four kickers (K1 to K4) are located in the arcs, two on either side of the injection point. The septum S3 is located at the end of the straight section where TL2 connects with the storage ring.

Published by the American Physical Society under the terms of the Creative Commons Attribution 4.0 International license. Further distribution of this work must maintain attribution to the author(s) and the published article's title, journal citation, and DOI.
Besides these pulsed elements, the injection cells layout and optics functions are identical to the ones of the standard cells. This preserves the 16-fold lattice symmetry and maintains optimal lifetime and dynamic aperture. As a result, sextupole magnets are located in between the injection kickers and the stored beam samples nonlinear fields when the injection bump is pulsing. Bump closure is calculated for its maximum amplitude by adjusting the field values of the four kickers. Due to the nonlinear fields of the sextupoles, this calculation does not hold for
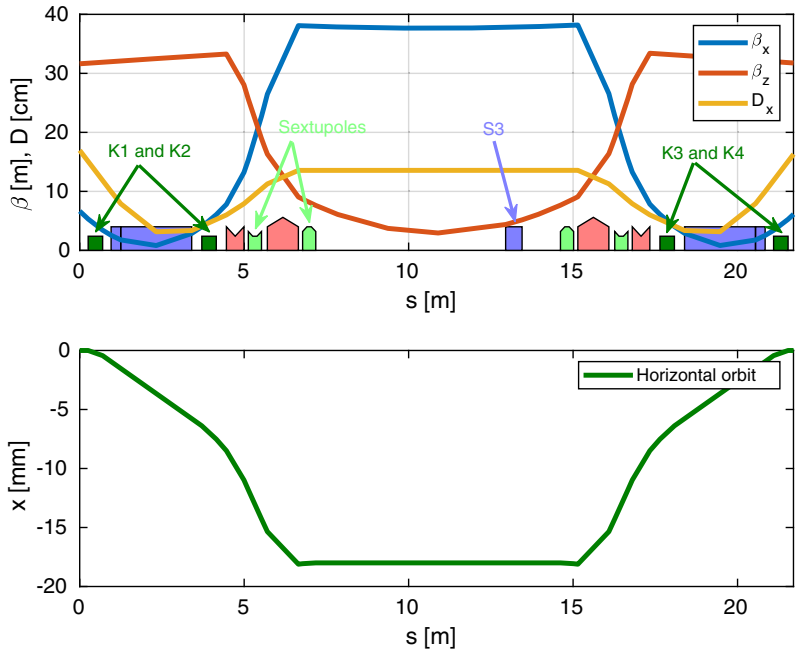

FIG. 1. Injection zone layout and optics functions (top). Closed orbit bump with nominal amplitude of $-18 \mathrm{~mm}$ (bottom). 
intermediate amplitudes and transient orbit distortions are observed during injections when the bump is ramped up and down. These orbit distortions were found to be the dominant source of injection perturbations due to the injection systems. While injection perturbations were not considered as an issue in decay mode (two injections per day), with the introduction of top-up operation and injections every 20 minutes a significant reduction was required to allow beam line users to acquire data continuously.

\section{SOURCES OF PERTURBATION}

The ESRF beam line scientists require a pollution, defined as the ratio between electrons present in the unfilled bucket and the ones present in the filled buckets, as low as $10^{-10}$. The cleaning of unfilled buckets is performed using a resonant excitation sending the unwanted electrons onto a scraper. Bunch cleaning was initially performed in the storage ring introducing unacceptable emittance growth during injections. Although not related to the injection systems this emittance growth was dominating the overall perturbation during top-up. Empty buckets are populated during the production and acceleration of electrons in the linac. A similar system was therefore installed in the booster to suppress all disturbances on the stored beam related to bunch cleaning. A detailed description of the system is provided in Ref. [2].

Static errors or slow perturbations on the storage ring lattice and orbit can generally be compensated using dedicated correctors and feedback loops. Injection perturbations are however driven by fast pulsing magnets and cannot be compensated by standard methods. The magnets located close to or in the storage ring will contribute most to the overall effect. Starting from the booster extraction towards the storage ring, the first perturbing elements are the TL2 dipole magnets, and most significantly the last (D5). These magnets are powered by the booster power supply system and pulsed at the booster cycling frequency ( $1 \mathrm{~Hz}$ or $10 \mathrm{~Hz}$ for the old power supply system and $4 \mathrm{~Hz}$ for the new one operated since 2017). These perturbations have sufficiently low frequency to be cured by the fast orbit feedback (FOFB). In addition, a shielding box was installed around D5 to minimize these perturbations at the time the feedback was not operational. The next elements are the TL2 in-air septa $S 1 / 2$ connected in series to a power supply generating a $2 \mathrm{~ms}$ half-sine pulse for a total deflecting angle of approximately $90 \mathrm{mrad}$. These are followed by the invacuum septum $\mathrm{S} 3$ connected to a power supply generating a $66 \mu$ s half-sine pulse for a total angle of $23 \mathrm{mrad}$. The last perturbing elements are the four injection kickers located in the storage ring. They are individually powered and give a kick of 1-2 mrad each with a $3 \mu$ s long rectangular pulse shape corresponding to one storage ring turn.

The injection perturbations can be classified in two categories: (i) Indirect perturbations resulting from fringe fields or eddy currents. Although some effect is expected

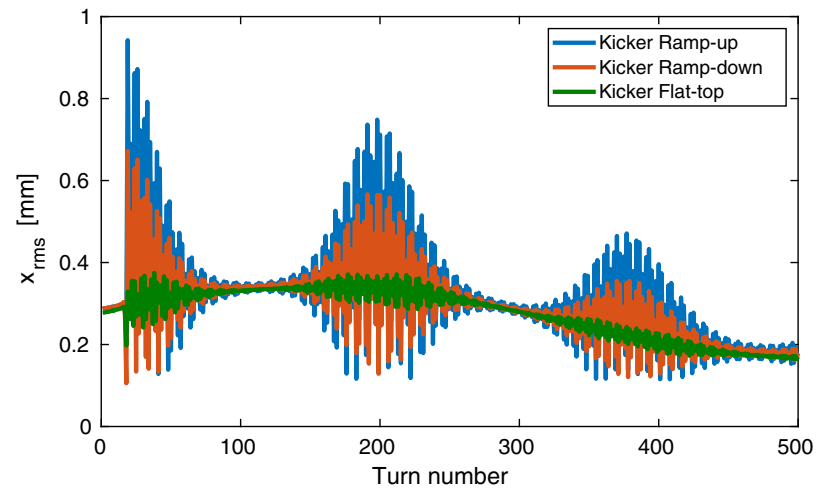

FIG. 2. Injection perturbation without compensation.

from uneven coating of the ceramic chambers these are dominated by septum magnets and typically have a longer timescale. (ii) Direct perturbations from the injection bump nonclosure. These have a much faster timescale and are comparable to a single kick. Bunches will experience different kick amplitudes depending on their position in the bunch train. Nonclosure is mainly due to differences between the pulsers of the four kickers (timing, amplitude, flatness), mis-steering of one or more kickers and the sextupoles located inside the injection bump.

Figure 2 shows the horizontal perturbations observed prior to any compensation work. These data were recorded using the turn-by-turn BPM acquisition system. The acquisition window corresponds to one third of the machine $(1 \mu \mathrm{s})$. The positions measured by the BPMs correspond to the average of all bunches within this window. Single bunch data may therefore differ from these depending on their position in the bunch train. Each data point on Fig. 2 corresponds to the rms of all BPMs readings over one turn. The kickers are pulsed at turn number 18. The 500 turns following this initial excitation are shown. The ESRF is operating with relatively large chromaticities $Q_{x}^{\prime}=7$ and $Q_{y}^{\prime}=11$. A strong decoherence and a modulation of the envelop of the betatron oscillations by the synchrotron frequency are expected and explain the three wide peaks observed on the plot. Details on the theory of decoherence of betatron oscillations due to chromaticity can be found in Refs. $[3,4]$. It should be noted that any attempt to actively damp the dipole perturbation should be faster than the decoherence in order to be effective. The fast oscillations within each of these peaks correspond to the betatron oscillations triggered by the residual kick from the injection bump nonclosure. Betatron oscillations are vanishing with the same timescale as radiation damping (several milliseconds). These oscillations are performed around a slow orbit distortion mainly driven by the septa fringe fields and eddy currents induced on the S3 septum blade. The slow distortion is vanishing with a longer timescale and will be described in more detail in the following section. For this measurement the kicker pulse was divided in three segments: the flat-top, the ramp-up and the ramp-down. One third of the ring circumference was filled and the injection kickers timing 
was shifted by $\pm 1 \mu$ s to measure either of these segments. The bump closure is calculated on the flat-top. As seen on the green curve, some residuals remain that indicate imperfect closure. The stronger perturbation on the ramps is due to the sextupoles located inside the injection bump. The small asymmetry between the up and down ramps is due to differences between the pulse shapes of the kickers. In addition, ramping up the injection bump introduces a sudden change in path length. This will trigger energy oscillations at the synchrotron frequency. These were however found to be negligible for the users. In view of the diverse sources of perturbation and their characteristics, dedicated strategies were adopted to mitigate each of these contributions using existing systems and hardware.

\section{SEPTUM MAGNETS COMPENSATION}

When analyzing the septum magnets perturbation, two effects should be distinguished: (i) The initial exciting field and associated fringe field. They are in theory perfectly shielded, but in practice leaking onto the stored beam orbit. They result in closed orbit distortions with the same time structure as the pulse shape and are well described by theory. (ii) The resulting eddy currents. They depend on the frequency of the exciting pulse and the magnet environment. They can travel along beam pipes, survive for a significant amount of time and are more difficult to model.

In Ref. [5] these issues were approached separately and corrected at the source with a number of actions. At the ESRF it was decided to treat them as a whole and implement an active damping system. Even though the septum perturbation is relatively slow, the limited FOFB bandwidth does not allow for dynamic correction in feedback mode. The perturbation is however very reproducible and a feed-forward system can effectively be used. For this purpose, the FOFB was modified in order to play an arbitrary waveform when a trigger signal is issued. There is a unique waveform in each plane for the 14 correctors used in the fast feed-forward correction. On top of this waveform, a set of 14 coefficients defines the amplitude and sign of the strength sent to each of the corrector magnets. The perturbation is measured using the BPM system in turn-by-turn mode and is averaged over a few shots. This measurement is used to compute the ideal correction strength for the 14 correctors as well as the unique waveform using a singular value decomposition (SVD) inversion of the response matrix. Because the rf straight section is located immediately after the injection cells it is possible to use the correctors of more than two full cells to compensate for the septum perturbation without impacting any beam line source.

Figure 3 shows the perturbation introduced on the stored beam by the septum magnets $S 1 / 2$ and $\mathrm{S} 3$ before and after compensation. The impact of the fast orbit correction algorithm added on top of the compensation is also shown. The individual contributions of $\mathrm{S} 3$ and $S 1 / 2$ can be distinguished. The initial sharp excursion corresponds to
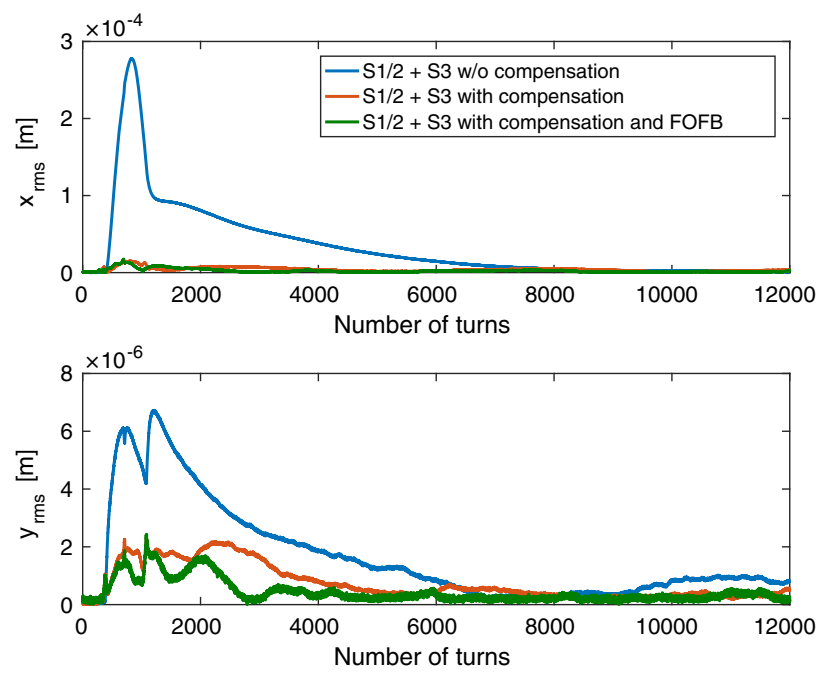

FIG. 3. Perturbation from septum magnets with and without compensation and FOFB.

the $S 1 / 22 \mathrm{~ms}$ (approximately 700 turns) pulse. Eddy currents induced by the $S 1 / 2$ pulse are rather limited and the perturbation rapidly vanishes together with the exciting field. While the S3 exciting field is very well shielded by the eddy currents circulating in its copper blade, when the exciting field is back at zero the eddy currents remain and are responsible for the very long tails seen on the perturbation. This much longer timescale corresponds to the time it takes for eddy currents to dissipate in copper under vacuum. The effect of the compensation is clearly visible and an improvement of almost a factor 20 is obtained in the horizontal plane. The residual peak rms orbit distortion is $15 \mu \mathrm{m}$. In the vertical plane, the initial perturbation is much smaller and the gain is only a factor 3 . The residual peak rms orbit distortion is a couple of microns. The fast orbit correction algorithm was activated while the compensation was running without significant signs of degradation.

Eddy currents circulating in the S3 septum blade can be suppressed using a full sine wave excitation instead of the presently used half-sine wave [6]. This option was considered and tested at ESRF on the present machine and gave approximately a factor 5 improvement. For technical reasons this solution was not implemented in the new inhouse S3 power supply but could be considered as a future development.

\section{KICKERS PERTURBATION}

The overall perturbation introduced by the kickers involves a certain number of processes. A description of these processes will be given in this section. The injection bump closure is calculated at its maximum amplitude, the pulse flat-top. This is done experimentally by adjusting the strengths of the four kickers in order to minimize the trajectory leakage on the first turn after the kickers are pulsed. Bump closure for intermediate amplitudes is 
maintained only if the ratio of the strengths of the kickers, $\beta$-functions, and phase advances between the kickers remain constant. This imposes strictly identical pulses for the four kickers and that no nonlinear element is located inside the bump.

At the ESRF, the lattice design imposes sextupoles inside the injection bump and the second constraint is not fulfilled. The sextupole nonlinear field will modify the trajectory as a function of the bump amplitude as follows:

$$
x_{1}^{\prime}=x_{0}^{\prime}-\frac{K_{2} L}{2} x_{0}^{2},
$$

where $x_{0,1}^{\prime}$ are the horizontal angles upstream and downstream the sextupole, $K_{2} L$ is the integrated sextupole strength, and $x_{0}$ is the horizontal offset, or bump amplitude at the sextupole. The kicker pulse extends over a complete revolution. Different dipole and quadrupole kicks are sampled by the bunches depending on their position in the train. The dipole perturbation profile along the bunch train consists of an unperturbed central part surrounded by two equal peaks. The central part corresponds to the kicker pulse flat-top for which the injection bump is closed. The peaks are related to the injection bump ramps for which the bump closure is perturbed by the nonlinear sextupoles fields. The gradient perturbation profile follows the kicker pulse and reaches a maximum at maximum bump amplitude.

This is illustrated in Fig. 4 where the perturbations were computed using an ideal model. The top plot shows the first turn horizontal rms trajectory due to dipole errors along the bunch train. The bottom plot shows the ratio between the nominal rms $\beta$-functions and the first turn perturbed $\beta$-functions due to gradient errors along the bunch train. The kicker pulse is shown for indication. Simulations were performed using the nominal $18 \mathrm{~mm}$ bump amplitude. Most of the time, the bump amplitude is set to lower values in order to avoid injection saturation when filling high current bunches. The simulated orbit perturbation reaches a maximum of almost $2 \mathrm{~mm}$ on the ramps of the kicker pulse. The rms $\beta$-function is increased at most by a factor 4
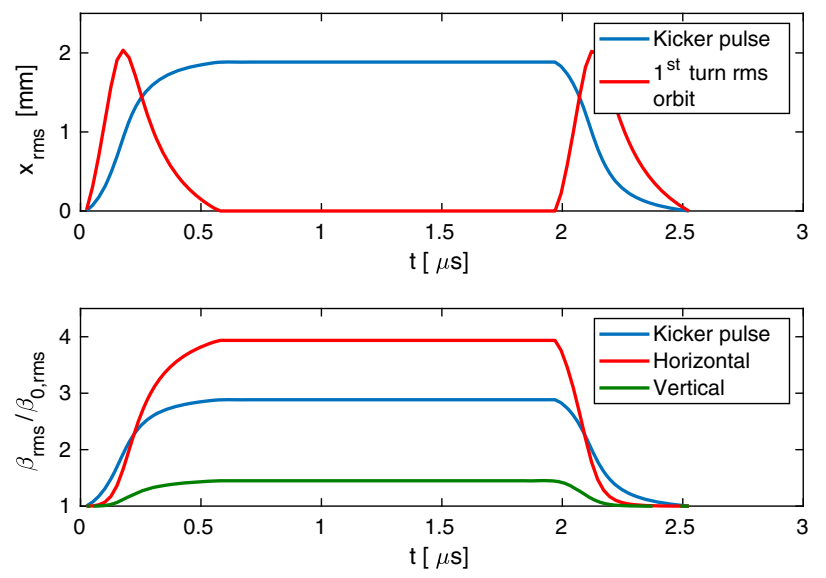

FIG. 4. rms orbit and $\beta$-function evolution over a kicker pulse. in the horizontal plane on the flat-top. These perturbations represent major disturbances to the beam line users during injection and compromise continuous data acquisition during top-up operation. Dipole errors can be corrected using standard orbit correction techniques. However, these cannot compensate for the additional quadrupole introduced by the off-axis sextupoles. Novel approaches for compensation were therefore specifically developed at ESRF and will be discussed in the next section.

The four kickers are powered independently. During the design phase, power supplies, cable lengths, and ceramic chambers metallic coating are specified to be identical. However, imperfections remain and generating four identical kicker pulses is not achievable. Nevertheless, a certain number of parameters such as delays, slope of the rise-time or properties of the power supplies electrical circuits are adjustable. Based on beam measurements, these can be empirically tuned to minimize discrepancies.

For this purpose, a dedicated diagnostic was developed to measure the perturbation profiles and characterize the bump closure along the bunch train. The position data are recorded using a BPM optimized for this application. The radio-frequency (rf) signals from a set of four capacitive pick-ups are recorded at a rate of 304 samples per turn using the analog digital convertors (ADC) of a Libera Sparks [7] electronics. These ADC recordings are downloaded and processed in order to provide position data with the bandwidth of a few MHz. This does not allow us to measure individual bunches but is sufficient to characterize the perturbation profile along the bunch train.

Figure 5 shows examples of perturbation profiles with mismatched kicker pulses measured with this dedicated diagnostic. The samples shown on this figure represent four consecutive turns recorded after the kickers are pulsed. The perturbation profile shown on the top plot of Fig. 4 modulated by betatron oscillations is expected in the ideal case. The strongly asymmetric amplitudes between the ramps seen

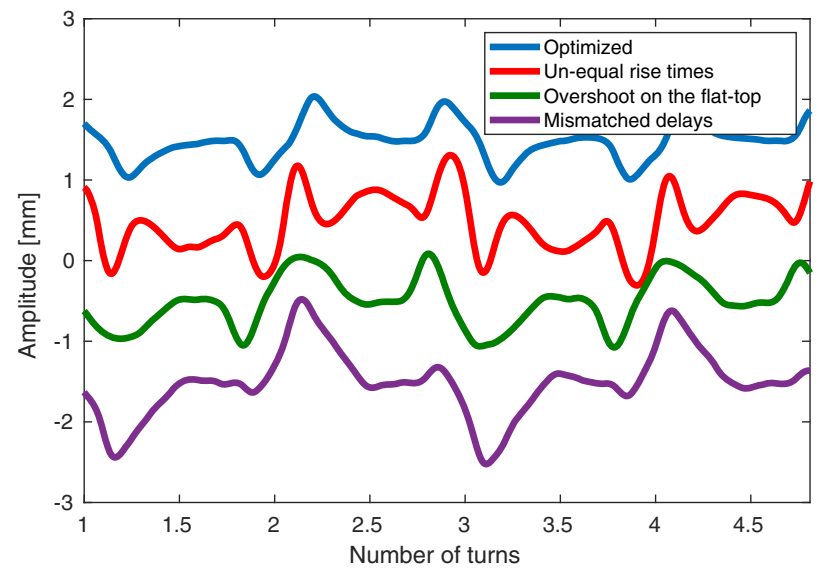

FIG. 5. Examples of perturbation profiles observed with mismatched kicker pulses, the curves are voluntarily shifted vertically for visibility. 
on the bottom curve are symptomatic mismatched delays. Perturbation peaks with different width as seen on the green curve indicate a current overshoot on the flat-top. This was solved by a change of capacity in one kicker to flatten its pulse shape. The red curve is the result of strongly mismatched inductances, and consequently rise-times as seen by the sharp perturbation on the ramps and the symmetrically perturbed flat-top. Finally the blue curve is the optimized case, which is close to theoretical expectations. All these quantities can suffer slow drifts and regular monitoring is in charge to maintain high performance levels. Random fluctuations cannot be avoided and were estimated to contribute for approximately $6 \%$ of the total perturbation.

\section{KICKERS PASSIVE COMPENSATIONS}

Similar issues, related to sextupoles inside the injection bump, were faced and solved in the past at the Spring8 synchrotron [8] by using specific sextupole settings. This method was tried at ESRF but led to an unacceptable lifetime loss [9] and was therefore abandoned. For the same reasons it was not possible to turn off or remove these sextupoles from the lattice. Several alternative methods were developed at ESRF to passively correct this perturbation at the source.

\section{A. Nonlinear kickers using eddy current screens}

The perturbing sextupole field can be exactly canceled using opposite sign sextupoles at the same location. These would have to be pulsed to maintain lifetime between injections. Implementing new pulsed nonlinear elements was found technically impractical, complicated to operate reliably and required significant development time. A simpler alternative is to shape the field of the existing injection kickers $\mathrm{K} 2$ and $\mathrm{K} 3$, that see the large bump amplitude, to partially cancel the perturbation introduced by the sextupoles. The initial idea to produce the desired kicker field profile was to significantly misalign them and inject into the fringe field. Due to mechanical and power supply constraints the optimal position could not be reached. This resulted in an unacceptable degradation of the injection efficiency. A less invasive solution was then adopted. It consists of adding copper plates inside the kickers ferrite gap. When the kickers are pulsing eddy currents will start to circulate in these conductors, effectively shielding the electron beam from the kicker field.

The ESRF injection kickers are c-shape ferrite pulsed magnets [1] with titanium coated ceramic vacuum chambers. There is sufficient space between the ferrite gap and the vacuum chamber to slide $1 \mathrm{~mm}$ thick copper plates and leave enough margin for vertical alignment. The copper plates are glued onto the ferrite, paying special attention that no short circuit is introduced in the process. As shown in Fig. 6, the copper plates are precisely aligned with respect to the stored and bumped beams. When the

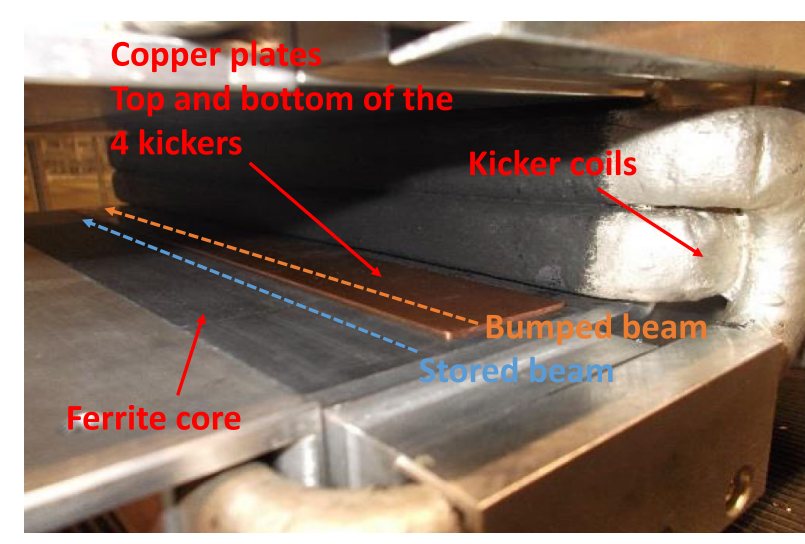

FIG. 6. Injection kicker equipped with copper plates.

injection bump is ramped up the electron beam is moved toward the plates and effectively samples a decreasing field. The current circulating in the kicker coils has to be increased accordingly to maintain the bump amplitude. In order to equalize the inductances, and hence the rise time of the four kickers, the copper plates were installed on all of them. However, for K1 and K4, the first and last kickers, the electron beam amplitude is not changing and the copper plates were placed such that the field attenuation is minimized on the beam path.

Figure 7 shows the horizontal field profile applied to K3 and the associated error with respect to an ideally compensated bump of $18 \mathrm{~mm}$. The same profile is applied to $\mathrm{K} 2$. Calculations were performed with COMSOL [10] using a 2D model of the kickers. Increasing the width of the copper plate $w$ is equivalent to approaching its edge to the stored beam. This results in higher attenuation on the bumped beam and on the stored beam at zero amplitude. Simulations predict a reduction of a factor 3.2 of the perturbation with optimized copper plates configuration and for the full $18 \mathrm{~mm}$ bump amplitude. Simple plates were first installed as shown in Fig. 6. Later on, more efficient c-shaped copper plates were installed on all four kickers.
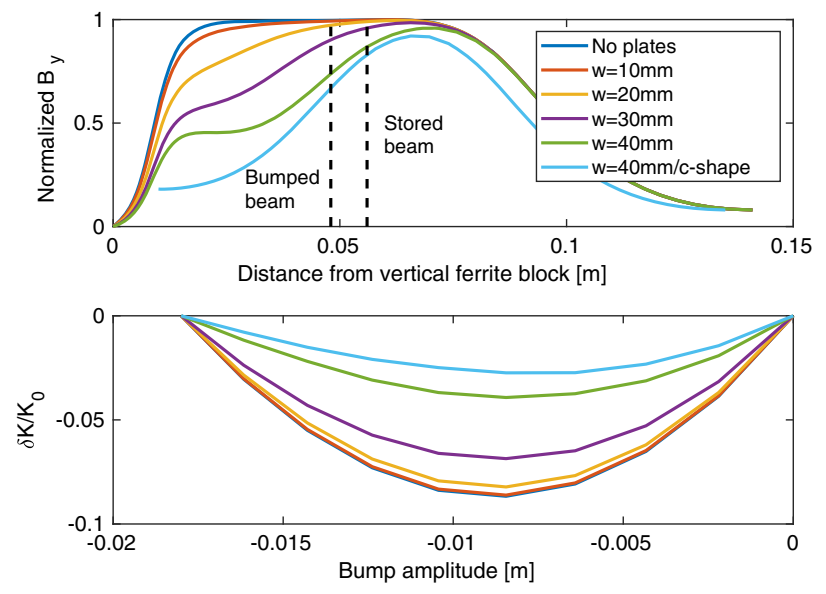

FIG. 7. K3 kicker field profile (top) and difference with ideally compensated perturbation for several copper plates of width $w$. 

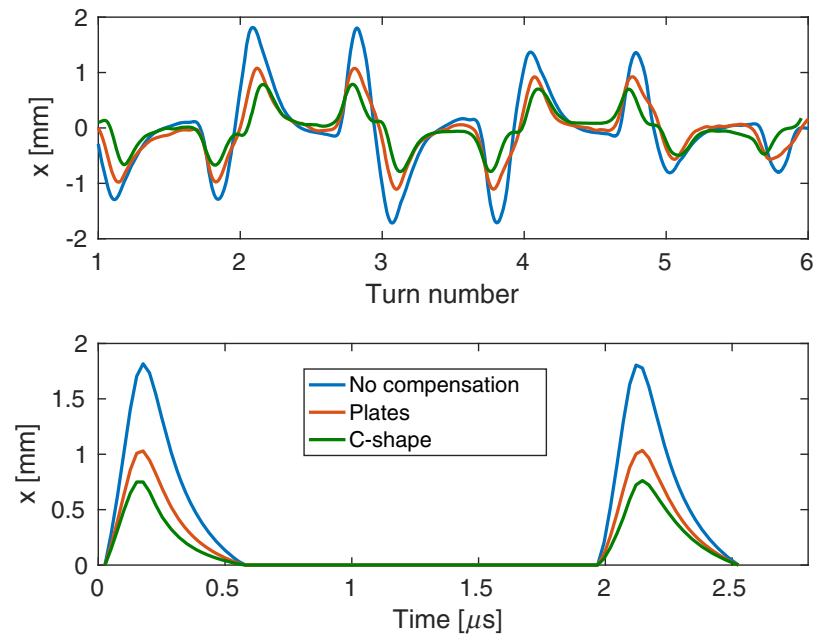

FIG. 8. Measured (top) and simulated (bottom) perturbation with and without compensation.

Figure 8 shows the measured and simulated perturbation with and without compensation using either simple or c-shaped copper plates. The simulations show one turn only. The phase is chosen to maximize the amplitude and the bump is perfectly closed on the flat-top. Measurements were performed at different times and kicker settings. Measurements with simple plates were not available for $16 \mathrm{~mm}$ bump amplitude, the standard operation value. In this case, the data were rescaled using the kicker K1 current as reference. In addition, the data without compensation were corrected for timing errors: the amplitudes of the two peaks were rescaled to their average value. Simulations and corrected data are in very good agreement. The perturbation is reduced by a factor 2.5 with c-shape copper plates and a bump amplitude of $16 \mathrm{~mm}$. For simple plates the perturbation is reduced by a factor 2.3 .

\section{B. Compensation of vertical perturbations}

The implementation of eddy current screens in the injection kickers also has an impact on the vertical plane. The vertical field is no longer purely dipolar and vertical kicker misalignments or beam offsets can potentially become severe sources of perturbations. After ensuring the kickers are aligned and the orbit is corrected, the residuals are corrected using a pair of skew quadrupoles. These skew quadrupoles, S4C3 and $\mathrm{S} 4 \mathrm{C} 4$, are located inside the injection bump, on either side of the injection point, where the beam experiences large horizontal excursions. When the bump is pulsing, the beam will experience vertical kicks proportional to this horizontal excursion. These can be used to locally correct for any vertical defects remaining in the kickers. A vertical bump will remain in the injection area with no impact on beam line sources.

Figure 9 shows an example of optimization of the skew quadrupole currents. S4C3 is located upstream of the injection point and $\mathrm{S} 4 \mathrm{C} 4$ downstream of the injection point.
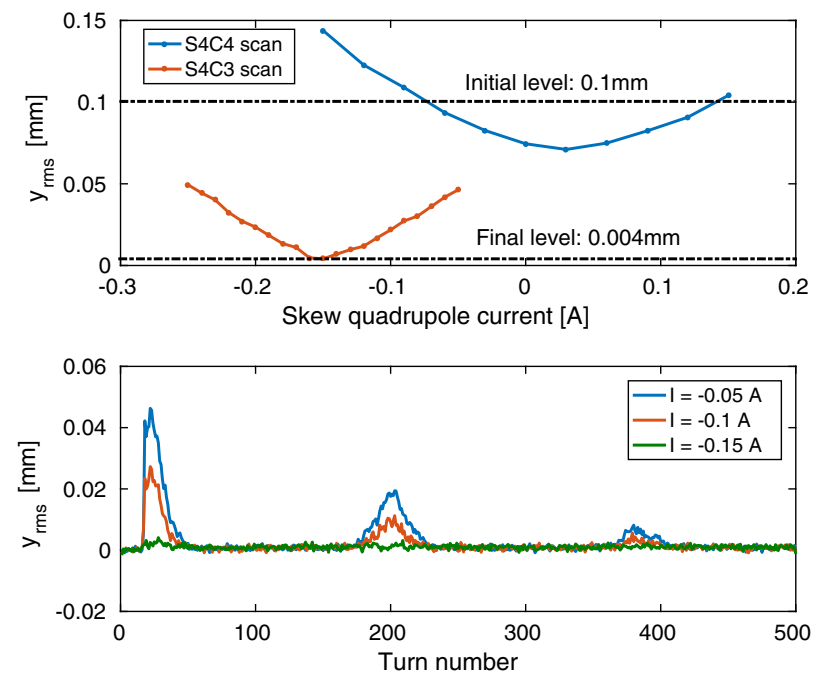

FIG. 9. Vertical perturbation minimization. Skew quadrupole (S4C3 and S4C4) current scans (top) and resulting rms orbit (bottom).

The final residual orbit is of the order of $4 \mu \mathrm{m}$. This is close to the resolution of the BPM turn-by-turn data as seen on the green curve of the bottom plot. In this case, the initial closed orbit was subtracted from the data and only the envelop of the oscillations is shown. Similarly to Fig. 2, the three peaks are due to decoherence introduced by high chromaticity. Optimal values can also be derived using SVD inversion. For operational simplicity, the skew quadrupoles are left $\mathrm{ON}$ all the time. This leads to a vertical emittance increase of a few picometers still remaining below the diffraction limit of $10 \mathrm{pm}$. The feasibility of ramping the skew quadrupoles during injection was demonstrated. Emittance fluctuations of a few picometers during injections was however seen as an additional source of perturbation and priority was given to transparent top-up. It should be noted that the compensation is most effective on the flat-top of the kicker pulse. Only a minor reduction was observed on the ramps. An active compensation system was therefore installed to correct the perturbation along the entire pulse. It is described in the following section of this paper.

\section{Compensation of quadrupolar oscillations}

As shown in Fig. 4, the sextupoles inside the injection bump are also the source of gradient errors. When the kickers are pulsed the beam will experience a quadrupole kick. Envelop oscillations will occur and will vanish with synchrotron damping. The effect is significant at full bump amplitude with an increase of almost a factor 4 of the horizontal $\mathrm{rms} \beta$-function over the first turn. As seen in Fig. 10, the sextupole field cancellation with nonlinear kickers reduces this effect by a factor 1.5 in the horizontal plane for a $16 \mathrm{~mm}$ bump amplitude. In the vertical plane the perturbation is almost reduced to zero on the flat-top leading to a significant improvement despite the degradation on the ramps. 


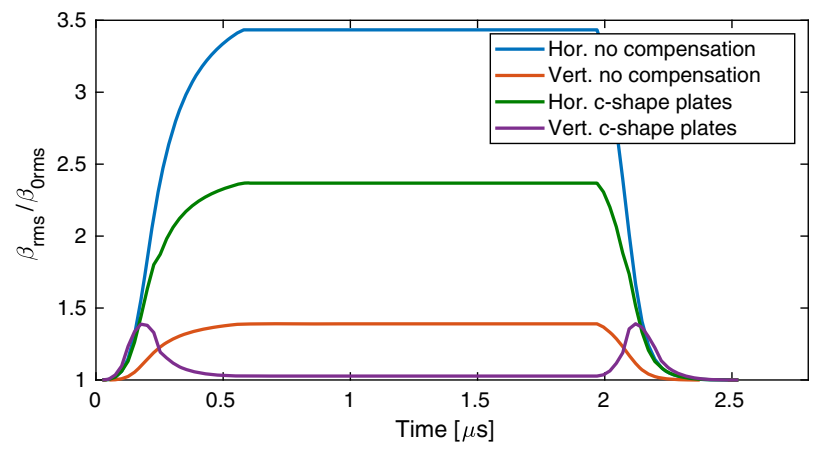

FIG. 10. rms $\beta$-function increase with and without eddy current screens.

In order to cancel the remaining gradient error an additional compensation was implemented. Multipoles of order $n$ have transverse field profiles with $x^{n-1}$ dependency. A combination of multipoles of different orders $n$ can therefore be used to cancel the gradient at a given amplitude without significantly affecting the field properties on axis. In the case of the ESRF, we need to cancel the gradient introduced by the sextupoles at the maximum bump amplitude of $18 \mathrm{~mm}$. This can be done using an octupole located in the injection straight section. The optimum octupole strength is given by the following system of equations:

$$
\begin{aligned}
x_{1}^{\prime} & =x_{0}^{\prime}-\frac{K_{2} L}{2 !} x_{0}^{2}-\frac{K_{3} L}{3 !} x_{0}^{3}, \\
\frac{\delta x_{1}^{\prime}}{\delta x} & =-K_{2} L x_{0}-\frac{K_{3} L}{2 !} x_{0}^{2}=0 .
\end{aligned}
$$

The values of $K_{2} L$ and $x_{0}$ are given by the sextupole settings and bump amplitude. The octupole strength is $K_{3} L$. The octupole introduces an additional angle $\Delta x^{\prime}=x_{1}^{\prime}-x_{0}^{\prime}$ on the injected beam. This needs to be compensated by the septum S3. These quantities can be analytically calculated as:

$$
\begin{aligned}
-\frac{K_{3} L}{2} & =\frac{K_{2} L}{x_{0}}, \\
\Delta x^{\prime} & =-\frac{K_{2} L}{6} x^{2} .
\end{aligned}
$$

Figure 11 illustrates how an octupole can be used to cancel the gradient introduced by a sextupole at large amplitude. A reduction of the dipole perturbation is also expected since the octupole reduces the total field on the side of the bump (positive on Fig. 11). These equations are exact in the case of a single sextupole compensated locally. The ESRF lattice includes several sextupoles at different phases. Full compensation will therefore not be achieved. The optimal octupole strength was therefore determined by tracking simulations and was found to be $K_{3} L=17.0 \mathrm{~m}^{-3}$.

Figure 12 shows the simulated reduction of dipole and quadrupole perturbation with the optimal octupole strength. The dipole perturbation is expected to be reduced by a

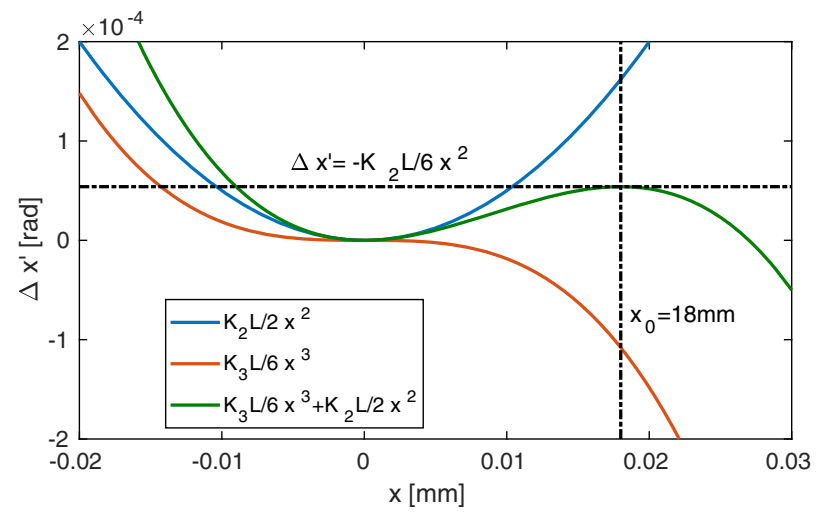

FIG. 11. Illustration of octupole compensation using $K_{2} L=$ $-1.0 \mathrm{~m}^{-2}$. The gradient is canceled at the maximum bump amplitude of $18 \mathrm{~mm}$.

factor 3 while the quadrupole perturbation is reduced by almost a factor 2. Providing the low integrated strength, it was decided to build this octupole in-house using spare refurbished insertion device permanent magnet blocks.

As seen in Fig. 13, where the Radia [11] model and simulated field are shown, the octupole is a c-shape magnet consisting of eight permanent magnet blocks. This allows for easy integration into the lattice. In order to compensate for the defect at the source it was placed immediately upstream the S3 septum, approximately at the center of the straight section. Because quadrupole oscillations are difficult to observe, the reduction of dipole oscillations before and after the octupole installation is used for experimental validation.

Figure 14 shows the measured injection perturbation before and after the octupole installation. An approximate factor 2 improvement is observed. This is 30\% lower than predictions and could indicate mechanical or magnetic
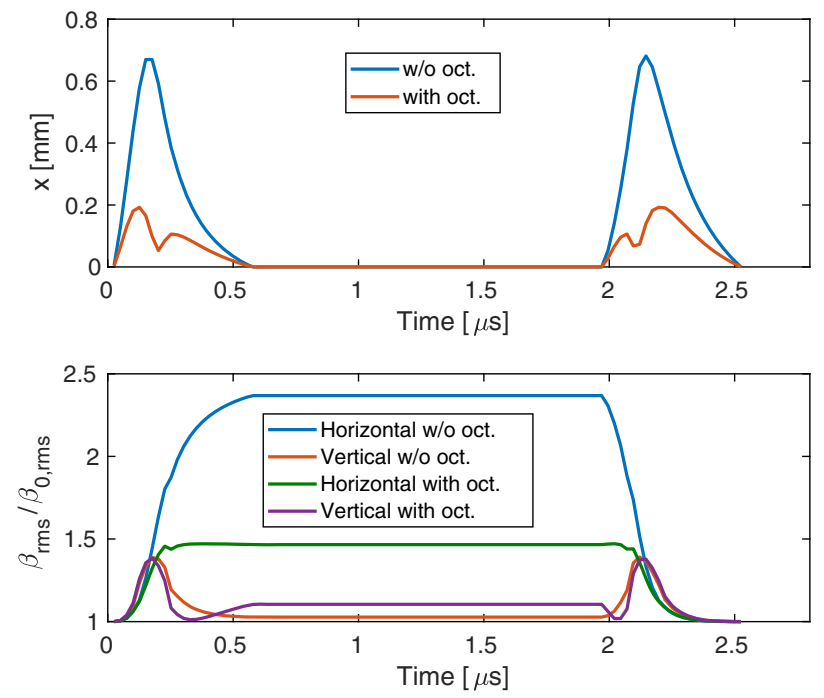

FIG. 12. Mitigation of quadrupole and dipole perturbation with the octupole. 


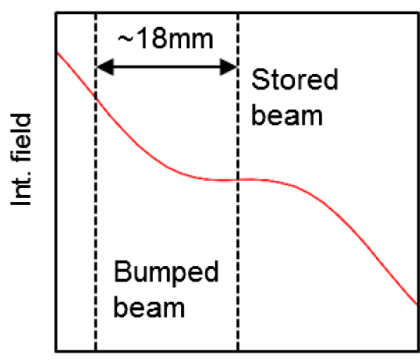

$X$ position

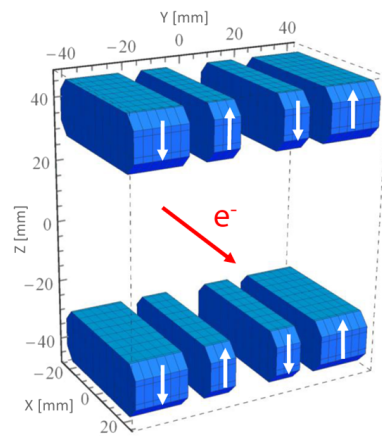

FIG. 13. Octupole Radia model and field calculation.
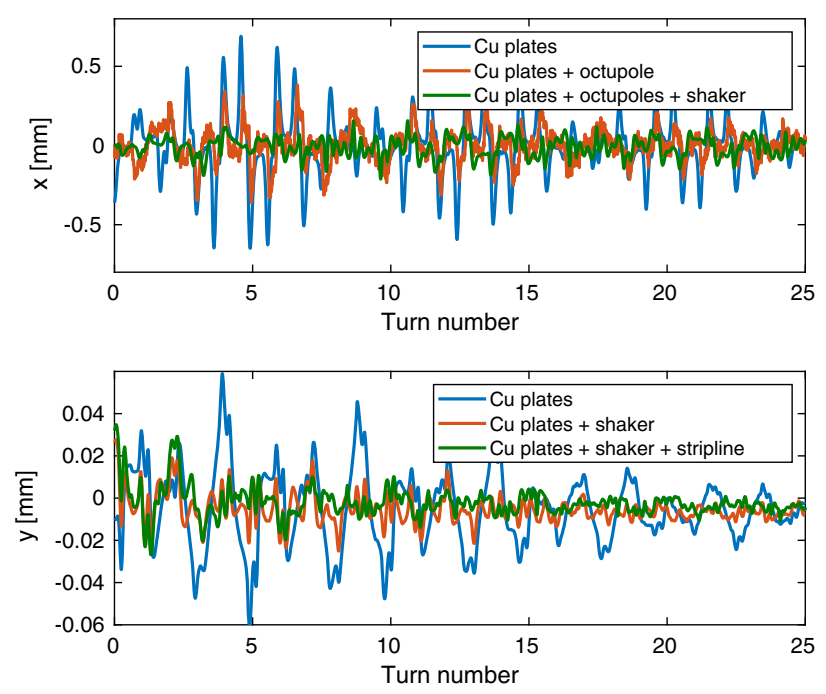

FIG. 14. Horizontal and vertical damping of injection perturbation using octupoles, shakers and the vertical stripline.

alignment errors or a bump amplitude difference with respect to the one used in simulations. Nevertheless, the improvement is significant without detrimental effects on injection efficiency or lifetime.

Combining multipoles to shape the field at a given amplitude could have further applications such as a tunable nonlinear kicker for transparent injection. The principle of using nonlinear kickers to provide transparent injection was introduced in Refs. [12,13]. This technique however suffers from the fact the injected beam often sees not only the desired dipole kick but also a strong gradient. This could potentially degrade the injection efficiency. Combining multipoles as proposed in this paper could help resolve this issue.

\section{KICKERS ACTIVE COMPENSATION}

Although significant improvements were achieved using passive techniques it was not possible to sufficiently reduce the perturbation such as to allow continuous data acquisition for the beam lines. The remaining perturbation is therefore damped with an active system.
The suppression of the perturbations would ideally require a corrector kicker located exactly at the phase of the perturbation, modulo $\pi$, or two kickers in order to produce a closed bump around the perturbation. However, the ESRF storage ring is equipped with a single wideband corrector per plane located at nonideal phases. The amplifiers that drive these correctors are not powerful enough to cancel the perturbation in one pass. This problem was mitigated by canceling the perturbation over a few beam revolutions: 5 turns for the vertical perturbation and 9 turns for the horizontal perturbation. The fractional tunes are 0.39 and 0.44 respectively. Over 5 turns the total vertical phase advance is approximately $4 \pi$. Over 9 turns the total horizontal phase advance is approximately $8 \pi$. Using this method we can easily adjust the phase of the correction signal. The required power on the amplifier is also significantly reduced since the correction angles sum up over several turns. The damping time of the perturbation is therefore approximately 5 or 9 turns depending on the plane. This is negligible compared to the transverse synchrotron damping time of several milliseconds. This system relies on the fact that the perturbation is well reproducible allowing for a feed-forward correction. The full system consists of one wideband dipole magnet per plane, the shakers described in Ref. [14], and a pick-up capable of measuring the bunches behaviour along the bunch train. The compensation is active only when the bump is pulsing.

The compensation is not perfect. This is mostly due to the finite bandwidth of the shakers not allowing the generation of high frequency field modulations. The ESRF is equipped with a vertical stripline kicker with larger bandwidth. This device is used to further improve the compensation in the vertical plane.

The results of the active compensation using shakers and the vertical stripline are shown in Fig. 14. In the horizontal plane, the perturbation is reduced by almost a factor 3 leading to a peak-to-peak perturbation of $200 \mu \mathrm{m}$. The perturbation damps to approximately $50 \mu \mathrm{m}$ peak in a few turns. In the vertical plane the peak perturbation is reduced by a factor 2 down to $30 \mu \mathrm{m}$. It damps to a few microns in a few turns.

\section{SUMMARY AND OUTLOOK}

Since the introduction of top-up and the request for transparent injection from the beam line users significant effort was made to reduce the injection perturbations at the ESRF. Diverse sources of perturbation require dedicated mitigation techniques. These can be summarized as follows: (i) cleaning of impurities performed in the booster; (ii) introduction of nonlinear kicker field to passively compensate for the sextupoles inside the bump; (iii) passive compensation of vertical perturbations using skew quadrupoles; (iv) passive compensation of quadrupole perturbations using an octupole in the injection straight; (v) active 


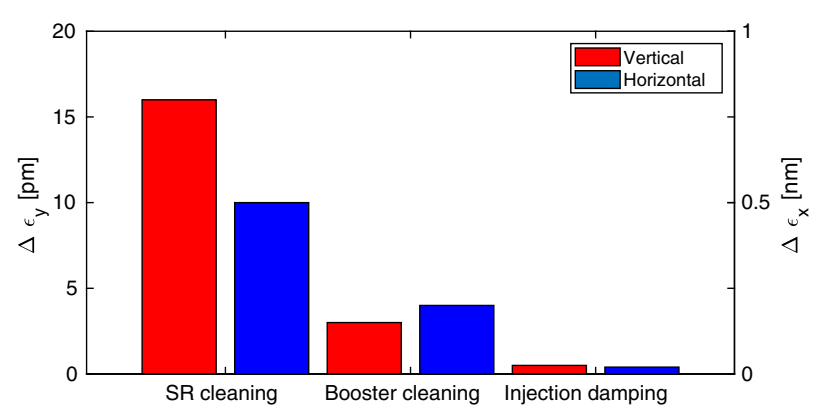

FIG. 15. Integrated vertical emittance growth during injection.

compensation of orbit distortions from septa using the FOFB in feed-forward mode; (vi) active compensation of kickers perturbations based on a feed-forward loop using shakers and a vertical stripline.

For its last physics runs, the ESRF was running with all these systems operational allowing for continuous data acquisitions and better thermal stability for the beam lines in all modes. The horizontal residual oscillation amplitude is of the order of $1 \sigma$ and the vertical residuals of the order of $2.5 \sigma$, both damping to a fraction of beam $\sigma$ in a few turns.

Figure 15 shows the horizontal and vertical emittance increase during injection measured by pinhole cameras and in-air x-ray monitors [15]. This represents an effective integrated emittance including all perturbations. In the horizontal plane, the values depend strongly on the bunch current, machine tuning and filling mode. Horizontal emittance increase of several nanometers were observed in some cases. The values shown here represent a lower limit. The cleaning in the storage ring (SR) was clearly the leading source of perturbation contributing for approximately $80 \%$ of the total increase in the vertical plane. After the implementation of all mitigation techniques (noted injection damping on the plot) presented above the residual increase in the vertical plane is $0.5 \mathrm{pm}(5 \%-10 \%$ increase depending on the mode and conditions) dominated by the kickers perturbations. In the horizontal plane, the increase is within the measurement noise. It is estimated to be less than $0.02 \mathrm{~nm}$ (approximately $0.5 \%$ ) when all system are optimized and running. Again, this horizontal increase depends on machine tuning and beam parameters. Values as high as $0.1 \mathrm{~nm}$ were observed in some cases.

The ESRF is presently undergoing an upgrade program (ESRF-EBS) which aims to reduce the horizontal beam emittance from $4000 \mathrm{pm}$ to $132 \mathrm{pm}$. Although the present situation does not impact users physics and data acquisition the smaller beam emittance will result in perturbations normalized to the beam size about 10 times larger than the present level. This is partly compensated by the absence of strong sextupoles inside the injection bump in the new lattice design. Nevertheless, alternative techniques and novel methods such as Refs. [12,16,17] are presently studied in order to maintain the present performance.

\section{ACKNOWLEDGMENTS}

The authors would like to thank the ESRF operation group and the injection/extraction unit, especially C. Richard, for their support during beam experiments and interventions with nonlinear kickers. A special thanks goes to B. Ogier for the mechanical design of the permanent magnet octupole and its support and their installation in the storage ring tunnel.

[1] ESRF foundation phase report (Red book), (1987).

[2] E. Plouviez, L. Farvacque, J. Koch, T. Perron, B. Roche, K. Scheidt, R. Versteegen, and S. White, Cleaning of parasitic bunches for timed structure filling of the ESRF storage ring during top-up operation, Proceedings of the 8th International Particle Accelerator Conference (IPAC 2017), Copenhagen, Denmark, 2017 (European Synchrotron Radiation Facility (ESRF), Grenoble, 2017).

[3] J. Shi and S. Ohnuma, Decoherence and recoherence of beam in phase space, in Proceedings of the 15th Particle Accelerator Conference, PAC-1993, Washington, DC, 1993 (IEEE, New York, 1993).

[4] I. C. Hsu, The decoherence and recoherence of the betatron oscillation signal and an application, Part. Accel. 34, 43 (1989).

[5] P. Lebasque, A. Loulergue, R. Ben El Fekih, J. P. Lavieville, M. Bol, D. Muller, and J. L. Marlats, Improvement on pulsed magnetic systems at SOLEIL, in Proceedings of the 9th European Particle Accelerator Conference, Lucerne, 2004 (EPS-AG, Lucerne, 2004), http://accelconf.web.cern.ch/ AccelConf/e04/.

[6] B. K. Kang and J. E. Milburn, Scaling law for diffused mangetic field in an eddy current passive septum magnet, Nucl. Instrum. Methods Phys. Res., Sect. A 385, 6 (1997).

[7] M. Znidarcic, E. Janezic, and M. Cargnelutti, Compact single pass BPM, Proceedings of the 6th International Particle Accelerator Conference (IPAC 2015), Richmond, Virginia, USA, 2015 (2015).

[8] H. Tanaka, T. Ohshima, K. Soutome, and M. Takao, Suppression of injection bump leakage caused by sextupole magnets within a bump orbit, Nucl. Instrum. Methods Phys. Res., Sect. A 539, 547 (2005).

[9] L. Farvacque (private communication).

[10] COMSOL, Comsol multiphysics reference manual, version 5.3, Inc., www.comsol.com.

[11] O. Chubar, P. Elleaume, and J. Chavanne, A 3D magnetostatics computer code for insertion devices, J. Synchrotron Radiat. 5, 481 (1998).

[12] K. Harada, Y. Kobayashi, T. Miyajima, and S. Nagahashi, New injection scheme using a pulsed quadrupole magnet in electron storage rings, Phys. Rev. ST Accel. Beams 10, 123501 (2007).

[13] T. Atkinson, M. Dirsat, O. Dressler, and P. Kuske, Development of a non-linear kicker system to facilitate a new injection scheme for the BESSY II storage ring, in Proceedings of the 2nd International Particle Accelerator Conference, San Sebastián, Spain (EPS-AG, Spain, 2011). 
[14] E. Plouviez and F. Loyer, ESRF synchrotron injectors tune measurement system, Proceedings of the 3rd European Particle Accelerator Conference (EPAC 1992), Berlin, Germany, 1992 (1992).

[15] B. K. Scheidt, Measurement of vertical emittance with a system of six in-air x-ray projection monitors at the ESRF, Proceedings of the 6th European Workshop on Beam Diagnostics and Instrumentation for Particle Accelerators, DIPAC2007, Venice, Italy, 2007 (2007).
[16] M. Aiba, M. Böge, F. Marcellini, Á. Saá Hernández, and A. Streun, Longitudinal injection scheme using short pulse kicker for small aperture electron storage rings, Phys. Rev. ST Accel. Beams 18, 020701 (2015).

[17] S. C. Leemann, Pulsed sextupole injection for Sweden's new light source MAX IV, Phys. Rev. ST Accel. Beams 15, 050705 (2012). 